\title{
Brillouin scattering and tribological study of $\mathrm{PMMA} / \mathrm{SiO}_{2}$ nanocomposite
}

\author{
D. Rouxel ${ }^{1,2}$, J. Eschbach ${ }^{1,2}$, B. Vincent ${ }^{1,2}$, G. Baret $^{3}$ \\ ${ }^{1}$ Laboratoire de Physique des Milieux Ionisés et Applications, Nancy Université, France \\ ${ }^{2}$ Laboratoire Européen de Recherche Universitaire Saarland-Lorraine-Luxembourg \\ ${ }^{3}$ DGTec, 178 rue de Mayoussard-38430 Moirans, France \\ (present adress : Gritche Technologies, 26 rue de Laffemas - 26000 Valence, France) \\ didier.rouxel@1pmi.uhp-nancy.fr
}

In recent years, nanocomposites materials, and in particular polymer nanocomposites have been developped for various applications, as high mechanical strengh, enhanced tribological properties and fire retardency [1-5]. These new materials could be used for extreme conditions in many industrials fields. Indeed, nanocomposites may combine the beneficial properties of polymer (easy shaping and low cost processing), with those of inorganic particles. The nanofillers may allow for tailoring the material according to the required properties (mechanical, tribological, optical, dielectric, etc.). In particular, due to the possible wide interphases between polymer and nanoparticles, some of the mechanical properties may be highly enhanced as fracture toughness or impact strength. Tribological applications could also potentially benefit from the development of polymer-based nanocomposites used as coatings. Therefore, some nanocomposites are beginning to be used as sliding elements in bulk or coating forms $[2,5]$.

In this work, we study $\mathrm{PMMA} / \mathrm{SiO}_{2}$ nanocomposites, prepared for industrial applications. Our analysis deals with friction and wear tests on the one hand and with Brillouin spectroscopy (BS) on the other. The latter is used to assess the exact elastic coefficient of the materials, which it has already been used for with materials ranging from crystalline to amorphous, including composites [6-10].

One of the main objectives of building such polymer-based nanocomposites is to enhance the wear performance of the polymer matrix. However, to obtain this effect, it is necessary to have a well incorporated powder, which means that the powder needs to be embedded into the matrix with efficient chemical bonds. In our case, silanisation was performed in order to improve the ability of the particles to react with the matrix. Therefore, in this study, the effects of the concentration of $\mathrm{SiO}_{2}$ nanoparticles and their surface treatment on the elastic properties, friction, and wear behaviour were investigated. Two kinds of samples were compared, with silanised $\mathrm{SiO}_{2}$ fillers and with non- silanised $\mathrm{SiO}_{2}$ fillers.

The nanoparticles used to prepare the nanocomposites are $\mathrm{SiO}_{2}$ nanoceramic synthesized by the company DGTec through precipitation from tetraethyl orthosilicate (TEOS). This process allows DGTec to produce monosized ball-shaped nanoparticles in a choice of size ranging from $30 \mathrm{~nm}$ to $500 \mathrm{~nm}$. In this study, nanopowders of less than $200 \mathrm{~nm}$ size were used in pure or silanised form. After the drying of the powder, silanisation was carried out by DGTec using methoxy-silane: silane Z-6011 (3-Aminopropyltriethoxysilane, Dow Corning) was used on the dried powder and stabilized with a low temperature treatment $\left(70{ }^{\circ} \mathrm{C}\right)$.

Masterbatches of nanocomposite at $2 \mathrm{wt} \%$ were realised in a doublescrew extruder with pellets of pure PMMA. Two masterbatches were prepared, with non-silanised $\mathrm{SiO}_{2}$ and silanised $\mathrm{SiO}_{2}$. Other concentrations, i.e. $0.1,0.5$ and $1 \mathrm{wt} \%$, were obtained by the extrusion 
of a mixture of pure PMMA pellets and loaded pellets. The forming of the nanocomposite was obtained by injection molding. Samples, $10 \times 40 \mathrm{~mm}^{2}$ and $3 \mathrm{~mm}$ thick, were then cut and polished $(\mathrm{Ra}<0.1 \mu \mathrm{m})$ for both tribological and Brillouin experiments. In this paper, nanocomposites with silanised particles are noted as samples-S, and with non-silanised particles as samples-NS.

Friction and wear tests were carried out using a pin - on - disk tribometer from CSMInstruments. The sliding tests were made under the constant load of $1 \mathrm{~N}$, which was applied to the rotating specimen by a stationary pin. The latter is a chrome stainlesssteel $(60 \mathrm{HRC}$ harness) ball with $6.0 \mathrm{~mm}$ diameter and a surface finish of $0.02 \mu \mathrm{m} \mathrm{Ra}$. For the tests performed at the constant rotational speed of $100 \mathrm{rpm}$, a circular wear track of $7 \mathrm{~mm}$ diameter was formed on the specimen surface. The analysis uses the final surface topography after 1000 rounds, in ambient air at a relative humidity of 45-55\%.

Brillouin spectroscopy (BS) is an optical method used for studying the hypersonic propagation ( $\mathrm{GHz}$ modes) in transparent liquid or solid media. In BS technique, it is not necessary to generate these modes externally, since thermal fluctuations result in density fluctuations that can be described as propagating hypersonic modes. Brillouin experiments study photons which are inelastically scattered by interacting with the hypersonic modes. The hypersonic modes are interpreted as acoustic phonons interacting with the incident photons of the laser beam. The laws of conservation of momentum $\left(\vec{k}_{i}\right.$ and $\vec{k}_{s}$ incident and scattered momentum) and energy ( $\hbar \omega_{\mathrm{i}}$ and $\hbar \omega_{\mathrm{s}}$ incident and scattered photon energy) determine the momentum $\vec{q}$ and the energy $\hbar \Omega$ associated to the acoustic phonon involved in the scattering process: $\vec{k}_{s}=\vec{k}_{i} \pm \vec{q}$ and $\hbar \omega_{\mathrm{s}}=\hbar \omega_{\mathrm{i}} \pm \hbar \Omega$. The frequency, $f=\omega / 2 \pi$, is found at the Brillouin peaks on the spectrum, $\mathrm{f}(\mathrm{q})=\Omega(\mathrm{q}) / 2 \pi$ being the frequency of the acoustic phonons that cause light scattering. Therefore, in our geometrical configuration, the acoustic velocity is obtained by the relation: $v(q)=\frac{2 \pi f(q)}{q}=\frac{f \lambda_{\text {laser }}}{\sqrt{2}}$ and the elastic constant $\mathrm{c}_{11}$ is determined from the equation $c_{11}=\rho v^{2}$, where $\rho$ is the mass density of the material.

For the experiments, the initial samples are polished on both sides before being analyzed in the $90 \mathrm{~A}$ configuration. Argon laser, Innova coherent, is used with a $\lambda=514.5 \mathrm{~nm}$ wave-length. The scattered light is analyzed in a 5-pass Fabry-Perot interferometer which produces the very high resolution needed for Brillouin spectroscopy. The signal is then amplified by a photo-multiplier and treated by a multi-channel analyzer. The acoustic phonon frequency shift is obtained from Brillouin spectra, and then $\mathrm{c}_{11}$ from the above equations.

Tablel presents the variation of the wear depth after 1000 rounds, for all the samples tested. In this work, wear is quantified by the average depth of the track after 1000 rounds. This average is calculated from the values obtained from ten profiles separated from each other by $12 \mu \mathrm{m}$. As can be observed in the table, compared to the case of $2 \mathrm{wt} \%$ silanised and non-silanised particles, the wear rate for pure PMMA is higher. The first result is then that pure PMMA shows the highest wear, compared with silanised and non-silanised particles, and for a concentration of nanoparticles up to $2 \mathrm{wt} \%$. The second is that, for the three concentrations compared $(0.1 \mathrm{wt} \%, 0.5 \mathrm{wt} \%$ and $2 \mathrm{wt} \%)$, nanocomposites with silanised particles always exhibit a better tribological behaviour than those with non-silanised particles. The resulting material has the properties that were expected. A third result is more 
surprising, related to the minimum of wear observed for the concentration of about $0.5 \mathrm{wt} \%$. Even if getting the precise value of the optimum concentration would require a smaller step between the samples, the presence of a minimum is at least clear for silanised $\mathrm{SiO}_{2}$.

Table 1: $\mathrm{c}_{11}$ and depth of wear track for silanised and non-silanised samples

\begin{tabular}{|l|l|l|}
\hline Sample & $\mathrm{c}_{11}(\mathrm{GPa})$ & Depth of wear track $(\mu \mathrm{m})$ \\
\hline PMMA & 9.10 & 2.25 \\
\hline PMMA/SiO2-NS 0.1wt\% & 9.03 & 2.22 \\
PMMA/SiO2-NS 0.5wt\% & 8.96 & 1.40 \\
PMMA/SiO2-NS 2wt\% & 8.99 & 1.73 \\
\hline PMMA/SiO2-S 0.1wt\% & 9.13 & 1.6 \\
PMMA/SiO2-S 0.5wt\% & 9.41 & 0.93 \\
PMMA/SiO2-S 1wt\% & 9.24 & 1.33 \\
PMMA/SiO2-S 2wt\% & 9.30 & 1.54 \\
\hline
\end{tabular}

Table 1 presents also the variation of the elastic constant $\mathrm{c}_{11}$ versus the concentration of particles in the matrix and their surface treatment, as obtained by Brillouin spectroscopy. In the case of non-silanised nanoparticles, $\mathrm{c}_{11}$ slowly decreases with concentration, especially for low concentrations (up to $0.5 \mathrm{wt} \%$ ). On the contrary, with silanised nanoparticles, $\mathrm{c}_{11}$ increases to a maximum at around $0.5 \mathrm{wt} \%$, and remains higher than $\mathrm{c}_{11}$ for pure PMMA.

This different behavior of $\mathrm{c}_{11}$ in the material may be related to the strong interfacial bonding between silanised nanoparticles and the matrix. Furthermore, the silanisation process leads to a better dispersion of the nanoparticles by decreasing the surface energy. The clusters of nanoparticles in the matrix are then smaller after mixing.

Experimentally, it can be observed that Brillouin spectroscopy allows a qualitative but sensitive evaluation of the dispersion efficiency of the nanoparticles in the polymer matrix.

One of the purposes of incorporating nanoparticles in polymer PMMA is to increase the surface's mechanical properties, in particular regarding scratch resistance. In this context, the two different evolutions of $\mathrm{c}_{11}$ show a competition between two phenomena: on one hand, nanoparticles are responsible for an increase of $\mathrm{c}_{11}$, due to the embedding of harder material in the polymer matrix. On the other hand, nanoparticles obstruct the polymerisation, thus preventing a complete curing, or disturb the polymer network, decreasing $\mathrm{c}_{11}$. In the case of non-silanised nanoparticles, which are supposed to have the worst affinity with the polymer matrix, the second phenomenon clearly has more influence up to $1 \mathrm{wt} \%$. For higher concentrations, the stabilisation of $\mathrm{c}_{11}$ shows a compensation of these two opposite effects.

Silanisation can induce two main advantages: first, the nanoparticles are better dispersed within the matrix because of weaker Van-der-Waals interaction between silanised surfaces; secondly, silanisation can also favour chemical bonds between the polymer matrix and the particles. That is why, for all nanoparticles concentrations, $\mathrm{c}_{11}$ is higher for silanised nanoparticles than for non-silanised ones. For the highest concentrations tested, 1 and $2 \mathrm{wt} \%$, the evolution of $c_{11}$ appears to be lower, as it was for non-silanised nanoparticles. It should be noted that silanised nanoparticles produce a greater value of $c_{11}$ than that found in pure PMMA, for all volume ratio.

The evolution of PMMA/SiO $2-\mathrm{S} \mathrm{c}_{11}$ and wear properties may also be compared. This behaviour seems consistent for homogeneous materials. In particular, a minimum of wear 
depth is measured for the observed maximum of elastic constant $\mathrm{c}_{11}$ at $0.5 \mathrm{wt} \%$, $\mathrm{i}$. e. the wear resistance is linked to the elastic modulus. However, with a larger volume ratio of nanoparticles, a less stiff matrix, due to presence of nanoparticles in the polymer network, counterbalances the advantages of the nanoparticles.

Even if the wear resistance is lower for $\mathrm{PMMA} / \mathrm{SiO}_{2}-\mathrm{NS}$ than that for silanised nanocomposite, it remains enhanced compared to pure PMMA, even though the value of $\mathrm{c}_{11}$ is lower than for pure PMMA. In this case, we can assume that the hard nanoparticles provide a wear protection to the matrix. As the nanoparticles are not bonded as well to the matrix in PMMA/SiO 2 -NS as they are in $\mathrm{PMMA} / \mathrm{SiO}_{2}-\mathrm{S}$, the matrix is weaker for the unsilanised nanocomposite and the beneficial effect of nanoparticles on wear resistance is lower.

To summarize, a maximum increase of elastic coefficient was observed for about $0.5 \mathrm{wt} \%$ of silanised nanoparticles. This corresponds to a maximum of wear attenuation with respect to pure PMMA. On the contrary, for non-silanised nanoparticles, the nanocomposite elastic constant decreases for content below $1 \mathrm{wt} \%$ and then stabilizes. Nevertheless, the wear resistance enhancement compared to pure PMMA was observed on both $\mathrm{PMMA} / \mathrm{SiO}_{2}$ nanocomposite types, with silanised or non silanised nanoparticles.

Our study suggests that when there are nanoparticles present, the enhancement of stiffness and wear resistance is in competition with a disturbance in the polymer network. Silanisation decreases the disturbance, providing a higher $\mathrm{c}_{11}$ and a higher wear resistance.

\section{Acknowledgments}

The authors want to thank Richard Kouitat and Jean-Pierre Arnould for the tribological analyses and their helpful discussions.

\section{References}

[1] Zhu J, Morgan A B, Lamelas F J, Wilkie C A. Fire properties of polystyrene-clay nanocomposites Chem. Mater. 2001; 13 (10) : 3774-3780

[2] Friedrich K, Zhang Z, Scharb A K. Effects of various fillers on the sliding wear of polymer composites. Composites Science and Technology 2005; 65 (15-16 SPEC. ISS.) : 2329-2343

[3] Pu Z , Mark J E, Jethmalani J M, Ford W T. Effects of Dispersion and Aggregation of Silica in the Reinforcement of Poly(methyl acrylate) Elastomers. Chem. Mater. 1997; 9 (11) : 2442-2447

[4] Wang Q, Xue J, Shen W, Xue Q. The effect of nanometer SiC filler on the tribological behavior of PEEK. Wear 1997; 209 (1-2) : 316-321

[5] Wetzel B, Haupert F, Zhang M Q. Epoxy nanocomposites with high mechanical and tribological performance. Composites Sci. Technol. 2003; 63 (14) : 2055-2067

[6] Vincent B, Krüger J K, Elmazria O, Bouvot L, Sanctuary R, Rouxel D, Alnot P.

Imaging of microwave-induced acoustic fields in LiNbO3 by high-performance Brillouin microscopy. J. of Phys. D: Appl. Phys. 2005; 38 : 2026-2030

[7] El Hakiki M, Eschbach J, Rouxel D, Vincent B, Vialle S, Krüger J K, Elmazria O, Alnot P. Ferroelectrics 2008 , in press

[8] Bactavatchalou R, Alnot P, Baller J, Kolle M, Müller U, Philipp M, Possart W, Rouxel D, Sanctuary R, Tschöpe A, Vergnat C, Wetzel B, Krüger J K. The generalized Cauchy relation: A probe for local structure in materials with isotropic symmetry. J. Phys.: Conf. Ser. 2006; 40: 111-117

[9] Krüger J K, Müller U, Bactavatchalou R, Mainka J, Gilow C., Possart W, Tschöpe A, Alnot P, Rouxel D, Sanctuary R, Wetzel B. The generalized Cauchy relation as an universal property of the amorphous state. J. de Phys. IV. 2005; 129: 45-49

[10] Eschbach J, Rouxel D, Vincent B, Mugnier Y, Galez C, Bourson P, Krüger J K, Elmazria O, Alnot P. Development and characterization of nanocomposite materials. Mat. Sci. and Eng. C 2007; 27: 1260-1264. 